\title{
Phase II Study of Induction Chemotherapy with Docetaxel, Capecitabine, and Cisplatin Plus Bevacizumab for Initially Unresectable Gastric Cancer with Invasion of Adjacent Organs or Paraaortic Lymph Node Metastasis
}

\section{Jwa Hoon Kim, MD' \\ Sook Ryun Park, MD, PhD' \\ Min-Hee Ryu, MD, PhD \\ Baek-Yeol Ryoo, MD, PhD' \\ Kyu-pyo Kim, MD, PhD' \\ Beom Su Kim, MD, PhD2 \\ Moon-Won Yoo, MD, PhD'2 \\ Jeong Hwan Yook, MD, PhD² \\ Byung Sik Kim, MD, PhD² \\ Jihun Kim, MD, PhD ${ }^{3}$ \\ Sun-Ju Byeon, MD, PhD 3 \\ Yoon-Koo Kang, MD, PhD'}

Departments of ${ }^{1}$ Oncology, ${ }^{2}$ Surgery, and ${ }^{3}$ Pathology, Asan Medical Center, University of Ulsan College of Medicine, Seoul, Korea
Correspondence: Yoon-Koo Kang, MD, PhD Department of Oncology, Asan Medical Center, University of Ulsan College of Medicine, 88 Olympic-ro 43-gil, Songpa-gu,

Seoul 05505, Korea

Tel: 82-2-3010-3230

Fax: 82-2-3010-8772

E-mail: ykkang@amc.seoul.kr

Received January 2, 2017

Accepted May 22, 2017

Published Online May 24, 2017

*Jwa Hoon Kim, Sook Ryun Park, and

Min-Hee Ryu contributed equally to this work.

\section{Purpose}

The purpose of this study was to evaluate the efficacy and safety of induction chemotherapy with docetaxel, capecitabine, and cisplatin (DXP) plus bevacizumab (BEV) on initially unresectable locally advanced gastric cancer (LAGC) or paraaortic lymph node (PAN) metastatic gastric cancer (GC).

\section{Materials and Methods}

Patients with LAGC or unresectable PAN metastatic GC received six induction chemotherapy cycles $\left(60 \mathrm{mg} / \mathrm{m}^{2}\right.$ docetaxel intravenously on day $1,937.5 \mathrm{mg} / \mathrm{m}^{2}$ capecitabine orally twice daily on days 1-14, $60 \mathrm{mg} / \mathrm{m}^{2}$ cisplatin intravenously on day 1 , and $7.5 \mathrm{mg} / \mathrm{kg}$ BEV intravenously on day 1 every 3 weeks), followed by conversion surgery. The primary endpoint was $\mathrm{RO}$ resection rate.

\section{Results}

Thirty-one patients with invasion to adjacent organs but without PAN metastasis $(n=14$, LAGC group) or with PAN metastasis regardless of invasion ( $n=17$, PAN group) were enrolled between July 2010 and December 2014. Twenty-seven patients (87.1\%) completed six chemotherapy cycles. The most common grade $\geq 3$ toxicities were neutropenia (71\%), neutropenia with fever/infection (22.6\%/3.2\%), and stomatitis (16.1\%). The clinical response and $\mathrm{RO}$ resection rates were $64.3 \%$ (95\% confidence interval [Cl], 46.6 to 82.0 ) and $64.5 \%$ (LAGC group, 71.4\%; PAN group, 58.8\%), respectively. The pathological complete regression rate was $12.9 \%$. After a median follow-up of 44.5 months (range, 39.4 to 49.7 months), the median progression-free survival and overall survival were 13.1 months ( $95 \% \mathrm{Cl}, 8.9$ to 17.3) and 38.6 months ( $95 \% \mathrm{Cl}, 22.0$ to 55.1$)$, respectively.

\section{Conclusion}

Induction chemotherapy with DXP+BEV displayed antitumor activities with encouraging RO resection rate and manageable toxicity profiles on patients with LAGC or PAN metastatic GC.
Key words

Stomach neoplasms, Induction chemotherapy, Gastrectomy 


\section{Introduction}

Stomach cancer is one of the leading causes of cancerrelated deaths in the world and Korea [1]. However, its 5 -year survival rate has increased as a result of early diagnosis and improved treatments for gastric cancer (GC) [1]. Furthermore, recent advances in chemotherapy for metastatic or recurrent GC treatment have contributed to the improvement in survival trends over time [2]. Additionally, multidisciplinary treatment approaches, including preoperative (induction), postoperative (adjuvant), and perioperative (induction plus adjuvant) therapies, have demonstrated significant survival benefits compared with surgery alone [3-7].

Although surgical resection remains the predominant curative treatment for GC, surgery is not usually utilized in the treatment of unresectable locally advanced gastric cancer (LAGC) or metastatic GC. However, the subgroups of patients with LAGC or metastatic GC are substantially heterogeneous in terms of disease burden or causes for noncurable status. Although palliative chemotherapy is the standard of care for such patients, systemic chemotherapy converts unresectable diseases to potentially resectable ones in a number of patients [8]. Considering that the achievement of curative surgery is the most critical prognostic factor in GC, the identification of candidates for potentially curative surgery and determination of the best treatment strategy for patients whose diseases were initially judged to be unresectable because of invasion to adjacent organs or distant metastasis are very important. Previous studies have suggested that induction chemotherapy followed by surgery could result in encouraging long-term outcomes in patients with unresectable LAGC or distant metastasis to one site [9-12], especially in the paraaortic lymph node (PAN).

Tumor shrinkage and downstaging, as a result of the induction chemotherapy's antitumor activity, are important factors to consider in increasing the likelihood of curative resection in patients with advanced gastric cancer (AGC). We previously reported that administration of induction chemotherapy with docetaxel, capecitabine, and cisplatin (DXP) led to curative surgery in a considerable proportion of patients with unresectable LAGC or PAN metastatic GC [12]. Prior studies have also demonstrated encouraging survival outcomes with the addition of bevacizumab (BEV, a monoclonal antibody against vascular endothelial growth factor) to various chemotherapeutic regimens used in metastatic GC treatment $[13,14]$. Although a randomized phase III Avastin in Gastric Cancer (AVAGAST) study failed to show a statistically significant overall survival (OS) benefit with the addition of BEV to chemotherapy as a first-line therapy for AGC treatment, this combination resulted in significantly increased progression-free survival (PFS) and overall res- ponse rate (ORR) [15]. The addition of BEV to induction chemotherapy should be considered to facilitate performance of conversion surgery with a curative intent.

We conducted a phase II trial of induction chemotherapy with DXP plus BEV to further improve the curative resection rate and long-term survival of patients with initially unresectable LAGC or PAN metastatic GC.

\section{Materials and Methods}

\section{Eligibility}

Patients with previously untreated, human epidermal growth factor receptor 2-negative, histologically proven gastric adenocarcinoma were considered eligible for inclusion in the study if they met the following criteria: aged between 18 and 70 years, 0-2 Eastern Cooperative Oncology Group performance status, adequate organ function, and initially unresectable GC caused by local invasion to adjacent organs (cT4b) or abdominal PAN metastasis, which is defined as more than $10 \mathrm{~mm}$ enlargement in the short-axis diameter on an abdominal/ pelvic computed tomography (CT) scan with uptake in an ${ }^{18} \mathrm{~F}$-fluoro-2-deoxy-d-glucose (FDG) positron emission tomography (PET) scan. We excluded patients with histological types other than adenocarcinoma, distant metastases in sites other than PAN, presence or history of other cancers, and definite ascites on a CT scan. The study protocol was approved by the Institutional Review Board of the Asan Medical Center. All procedures were followed in accordance with the ethical standards of the responsible committee on human experimentation (institutional and national) and the 1964 Helsinki Declaration and its later versions. Informed consent or its substitute was obtained from all patients before enrollment to the study (ClinicalTrials.gov identifier: NCT01471470).

\section{Treatment}

Based on the recommended doses determined in the previous studies, patients received $7.5 \mathrm{mg} / \mathrm{kg}$ BEV (Roche Co., Seoul, Korea) intravenously, $60 \mathrm{mg} / \mathrm{m}^{2}$ docetaxel intravenously, and $60 \mathrm{mg} / \mathrm{m}^{2}$ cisplatin intravenously on day 1 and $937.5 \mathrm{mg} / \mathrm{m}^{2}$ capecitabine orally twice daily on days 1-14 in a 3-week cycle. Prophylactic granulocyte colony-stimulating factor was not administered in this study. The patients received a total of six induction chemotherapy cycles with DXP plus BEV in cases where evidences of disease progression or intolerability were not observed. Subsequently, patients underwent surgery with the intention of performing 
a D2 lymphadenectomy or extended paraaortic lymphadenectomy within 4-6 weeks after the completion of induction chemotherapy. Surgeons performed extended paraaortic lymphadenectomy or PAN sampling if palpable PAN were present in the surgical field, whether or not there was enlargement of PAN on CT scan. For patients who initially experienced PAN metastasis, surgery was conducted only if the PAN decreased to less than $10 \mathrm{~mm}$ in the short-axis diameter on a CT scan or uptake in an FDG PET scan. Postoperative chemotherapy was administered depending on the surgical findings and decisions of the attending physicians. Patients who experienced disease progression during induction chemotherapy or had unresectable disease even after the completion of induction chemotherapy were subsequently administered with salvage chemotherapy.

\section{Evaluation}

The medical histories of all patients were obtained before treatment, including the physical examination, complete blood count (CBC) with differential, serum chemistry, electrolytes, coagulation, urinalysis, electrocardiogram, chest X-ray, abdominal/pelvic CT scan, and esophagogastroduodenoscopy. ${ }^{18} \mathrm{~F}-\mathrm{FDG}$ PET was performed in patients with more than $10 \mathrm{~mm}$ PAN enlargement in the short-axis diameter on a CT scan. During induction chemotherapy, $\mathrm{CBC}$ with differential was measured every week, whereas chemical and electrolyte assessments were conducted at each cycle. The presence of adverse events was evaluated prior to each chemotherapy cycle using the National Cancer Institute Common Toxicity Criteria ver. 3.0 [16]. Dose adjustment was performed based on the protocol. A CT scan was repeated every two cycles to evaluate the tumor response in accordance with the Response Evaluation Criteria in Solid Tumors ver. 1.0 [17]. Upon treatment completion, physical examination and blood tests, chest X-ray and CT scans, and esophagogastroduodenoscopy were performed every 3 months, every 6 months, and annually, respectively. The clinical and pathological stages were determined based on the seventh edition of the American Joint Committee on Cancer staging system. Furthermore, the histological data derived from patients who underwent gastrectomy were reviewed by two pathologists (J.H.K. and S.-J.B.). The tumor regression grades (TRG), which were determined using primary tumor specimens, were classified into the following five groups: TRG1 (absence of residual cancer cells with fibrosis extending through the wall), TRG2 (presence of few residual cancer cells scattered through the fibrosis), TRG3 (at least three residual tumor glands with predominant fibrosis), TRG4 (residual cancer outgrowing fibrosis), and TRG5 (absence of regressive changes) $[18,19]$. Pathological complete regression (pCR) was defined as TRG1 without tumor cells in the lymph nodes (LNs) $[18,19]$. Additionally, the pathological findings of the surgical or endoscopic biopsy tissues were examined.

\section{Statistical analysis}

The primary endpoint was the curative $\mathrm{R} 0$ resection rate, whereas the secondary endpoints were the ORR, pathological response, PFS, OS, and safety. The sample size was calculated using Fleming one-stage optimal design with the target curative resection rate. We enrolled 31 patients assuming that $P_{0}=0.5$ and $P_{1}=0.7$ with $\alpha=0.1$ (one-sided), $\beta=0.2$, and dropout rate equal to $10 \%$. The one sample $t$ test was used to assess the significance of the null hypothesis.

The OS was calculated from the first date of treatment to the date of death from any cause. Meanwhile, the PFS was calculated from the first date of treatment to the date of disease progression or death. PFS events were defined as disease progression during treatment with induction chemotherapy, recurrence after curative surgery, disease progression after noncurative surgery when at least stable disease was observed at the time of induction chemotherapy completion, and death. We estimated the OS and PFS using the Kaplan-Meier method and compared the survival outcomes between groups through the log-rank test. Discrete data were compared using Pearson's chi-square and Fisher exact tests, whereas quantitative data were compared using the MannWhitney U test. All tests were two sided, and $\mathrm{p}<0.05$ was considered statically significant.

\section{Results}

\section{Patient characteristics}

Thirty-one patients with initially unresectable GC were enrolled in the study between July 2010 and December 2014. The patients were categorized based on the cause of GC unresectability: LAGC group, which include patients with invasion to adjacent organs but without PAN metastasis $(\mathrm{n}=14)$, and PAN group, which include patients with PAN metastasis regardless of invasion to adjacent organs $(n=17)$. Sixteen patients $(51.6 \%)$ had pancreatic invasion with or without invasion to other organs, such as the colon or liver. Table 1 presents the baseline characteristics of the patients.

\section{Chemotherapy delivery and toxicity}

Patients were treated with a median of 6 cycles of induction chemotherapy with DXP+BEV (range, 1 to 6). Twenty-seven patients $(87.1 \%)$ completed a total of six induction chemother- 
Table 1. Baseline patient characteristics

\begin{tabular}{|cc|}
\hline Characteristic & No. $(\%)(\mathbf{n}=31)$ \\
\hline Age, median (range, yr) & $55(33-70)$ \\
Male sex & $26(83.9)$ \\
\hline ECOG PS & $2(6.5)$ \\
\hline 0 & $29(93.5)$ \\
\hline 1 & \\
\hline Primary tumor location & $2(6.5)$ \\
\hline Upper third & $13(41.9)$ \\
\hline Middle third & $13(41.9)$ \\
\hline Lower third & $3(9.7)$ \\
\hline Diffuse & \\
\hline Disease status & $14(45.2)$ \\
\hline Locally advanced T4b without M1 & \\
\hline (LAGC group) & $11(35.5)$ \\
\hline Pancreas invasion & $2(6.4)$ \\
\hline Pancreas+colon invasion & $1(3.2)$ \\
\hline Pancreas+liver invasion & $17(54.8)$ \\
\hline PAN metastasis (PAN group) & $13(41.9)$ \\
\hline PAN metastasis only & $2(6.5)$ \\
\hline PAN metastasis+pancreas invasion & $2(6.5)$ \\
\hline PAN metastasis+liver invasion & $24(77.4)$ \\
\hline Histology & $2(6.5)$ \\
\hline TA & $5(16.1)$ \\
\hline TA wet ring cell carcinoma & $28(90.3)$ \\
\hline Measurable disease & \\
\hline
\end{tabular}

ECOG PS, Eastern Cooperative Oncology Group performance status; LAGC, locally advanced gastric cancer; PAN, paraaortic lymph node; TA, tubular adenocarcinoma.

apy cycles. Meanwhile, chemotherapy was discontinued in four patients $(12.9 \%)$ due to gastric perforation $(n=2)$, death from sepsis $(\mathrm{n}=1)$, and tooth infection $(\mathrm{n}=1)$.

Of the total 175 cycles, the capecitabine, docetaxel, or cisplatin dose was reduced in 42 cycles $(24 \%)$ in 21 patients $(67.7 \%)$ primarily due to grade 3 febrile neutropenia $(n=7$, $22.6 \%$ ), grade $2 / 3$ oral mucositis $(n=7,22.6 \%)$, and grade 3 diarrhea $(n=2,6.5 \%)$. Moreover, chemotherapy administration was delayed in 12 cycles (6.9\%) in eight patients (25.8\%).

The median relative dose intensity for docetaxel, capecitabine, cisplatin, and BEV were 0.89, 0.74, 0.92, and 0.93, respectively. Table 2 summarizes the adverse events. The most common hematological adverse events with grade $\geq 3$ were neutropenia $(71.0 \%)$, infection with febrile neutropenia (candidemia-related sepsis, $22.6 \% / 3.2 \%$ ), and leukopenia (32.3\%). Moreover, the most common nonhematological adverse events with grade $\geq 3$ were stomatitis $(16.1 \%)$ and anorexia $(9.7 \%)$. Grade 3 gastric perforation $(9.7 \%)$, grade 3 pulmonary embolism $(3.2 \%)$, and grade 1 hypertension $(3.2 \%)$ were the adverse events considered to be associated with BEV. One treatment-related death caused by candidemia-associated sepsis was recorded.

Six out of the 20 patients who underwent $\mathrm{R} 0$ resection received a total of 24 adjuvant chemotherapy cycles (median, 4; range, 1 to 8 cycles), with the regimen consisting of fluoropyrimidine derivates $(\mathrm{n}=5)$ as well as docetaxel and cisplatin $(\mathrm{n}=1)$.

\section{Clinical responses}

Among the 31 patients, 28 had measurable lesions, of whom $18(64.3 \%)$ achieved partial response (PR) and $10(35.7 \%)$ had stable disease, which resulted in an ORR of $64.3 \%$ (95\% confidence interval [CI], 46.6 to 82.0 ). The disease control rate among all patients was 100\% given that the three patients with evaluable lesions had incomplete response or stable disease. Based on the CT scan results, clinical T, N, and TNM downstaging were observed in $22(71.0 \%), 28(90.3 \%)$, and $26(83.9 \%)$ patients, respectively. Table 3 displays the clinical stages in the LAGC and PAN groups following induction chemotherapy. Except for five patients, all experienced downstaging after induction chemotherapy.

\section{Surgical and pathological results}

Out of the 31 patients, 28 (90.3\%) underwent surgery, whereas three $(9.7 \%)$ did not because of surgery refusal $(n=1)$, death from sepsis $(n=1)$, and GC unresectability owing to persistent hepatoduodenal ligament invasion $(\mathrm{n}=1)$. Twenty-four patients $(77.4 \%)$ underwent D2 gastrectomy, of whom two patients with palpable PAN underwent additional extended paraaortic lymphadenectomy. Tumor cells were not detected in the resected PAN. Four patients $(14.3 \%)$ underwent only opening and closure due to unresectability caused by pancreatic invasion $(n=2,6.4 \%)$, severe adhesion $(n=1,3.2 \%)$, and peritoneal seeding $(n=1,3.2 \%)$. Curative resection was conducted in 20 patients, and the $\mathrm{R} 0$ resection rate was $64.5 \%$ (95\% CI, 47.7 to 81.3 ): $71.4 \%$ (95\% CI, 47.7 to 95.1 ) and $58.8 \%$ (95\% CI, 35.4 to 82.2 ) in the LAGC and PAN groups, respectively $(\mathrm{p}=0.707)$. The null hypothesis that the $\mathrm{R} 0$ resection rate was $50 \%$ was rejected $(\mathrm{p}=0.023)$. Four patients $(12.9 \%)$ achieved pCR, and the number of patients with TRG1, TRG2, TRG3, TRG4, and TRG5 were five (16.1\%), three (9.7\%), five (16.1\%), nine (29.0\%), and two (6.4\%), respectively. Among them, only one patient had residual tumor cells in the resected LNs. Table 4 shows the surgical and pathological results.

The surgical complications that occurred within 1 month after surgery included seroma $(n=2)$, hematoma $(n=1)$, tissue friability $(n=1)$, wound infection $(n=1)$, and adhesive ileus, which was the only complication that required intervention (adhesiolysis) ( $\mathrm{n}=1)$. 
Table 2. Adverse events (>5\%) and major adverse events $(<5 \%)$

\begin{tabular}{|c|c|c|}
\hline Adverse event & Grades 1-2 & Grades 3-4 \\
\hline \multicolumn{3}{|l|}{ Hematological } \\
\hline Leukopenia & $16(51.6)$ & $14(45.2)$ \\
\hline Neutropenia & $3(9.7)$ & $23(74.2)$ \\
\hline Anemia & $26(83.9)$ & $5(16.1)$ \\
\hline Thrombocytopenia & $20(64.6)$ & $1(3.2)$ \\
\hline \multicolumn{3}{|l|}{ Nonhematological } \\
\hline Febrile neutropenia & - & $7(22.6)$ \\
\hline Infection with neutropenia & 0 & $1(3.2)^{a)}$ \\
\hline Fatigue & $18(58.1)$ & $2(6.5)$ \\
\hline Anorexia & $24(77.4)$ & $3(9.7)$ \\
\hline Nausea & $16(51.6)$ & $1(3.2)$ \\
\hline Vomiting & $4(12.9)$ & $1(3.2)$ \\
\hline Stomatitis & $20(64.5)$ & $5(16.1)$ \\
\hline Diarrhea & $12(38.7)$ & $2(6.5)$ \\
\hline Alopecia & $29(93.5)$ & 0 \\
\hline Sensory neuropathy & $12(38.7)$ & 0 \\
\hline Nail changes & $14(45.2)$ & 0 \\
\hline Skin pigmentation & $18(58.1)$ & 0 \\
\hline HFS & $6(19.4)$ & 0 \\
\hline Abdominal pain & $6(19.4)$ & $2(6.5)$ \\
\hline Headache & $7(22.6)$ & 0 \\
\hline Epistaxis & $11(35.5)$ & 0 \\
\hline Constipation & $6(19.4)$ & 0 \\
\hline Sore throat & $3(9.7)$ & $1(3.2)$ \\
\hline Gastric perforation & 0 & $3(9.7)$ \\
\hline Thromboembolic event & 0 & $1(3.2)$ \\
\hline Hypertension & $1(3.2)$ & 0 \\
\hline Increased AST or ALT & $3(9.7)$ & $1(3.2)^{a)}$ \\
\hline
\end{tabular}

Values are presented as number (\%). HFS, hand-foot syndrome; AST, aspartate aminotransferase; ALT, alanine aminotransferase. ${ }^{\text {a) }}$ Grade 5: candidemia-related sepsis with multiorgan failure.

Table 3. Clinical downstaging after induction chemotherapy

\begin{tabular}{|c|c|c|c|}
\hline \multicolumn{2}{|c|}{ LAGC group ( $n=14,100 \%)$} & \multicolumn{2}{|c|}{ PAN group $(n=17,100 \%)$} \\
\hline Baseline clinical stage & $\begin{array}{c}\text { Clinical stage after } \\
\text { induction chemotherapy }\end{array}$ & Baseline clinical stage & $\begin{array}{l}\text { Clinical stage after } \\
\text { induction chemotherapy }\end{array}$ \\
\hline \multirow[t]{4}{*}{ Stage IIIB $(\mathrm{n}=7,50 \%)$} & Stage IB $(\mathrm{n}=2,14.3 \%)$ & Stage IV $(\mathrm{n}=17,100 \%)$ & Stage IIA $(n=2,11.8 \%)$ \\
\hline & Stage IIA $(n=1,7.1 \%)$ & & Stage IIB $(n=3,17.6 \%)$ \\
\hline & Stage IIB $(\mathrm{n}=3,21.4 \%)$ & & Stage IIIA $(n=5,29.4 \%)$ \\
\hline & Stage IIIB ( $\mathrm{n}=1,7.1 \%)$ & & Stage IIIB ( $\mathrm{n}=1,5.9 \%)$ \\
\hline \multirow[t]{3}{*}{ Stage IIIC $(\mathrm{n}=7,50 \%)$} & Stage IIA $(\mathrm{n}=2,14.3 \%)$ & & Stage IIIC $(n=2,11.8 \%)$ \\
\hline & Stage IIIA $(n=3,21.4 \%)$ & & Stage IV $(n=4,23.5 \%)$ \\
\hline & Stage IIIB $(n=2,14.3 \%)$ & & \\
\hline
\end{tabular}

LAGC, locally advanced gastric cancer; PAN, paraaortic lymph node. 
Table 4. Surgical and pathological results

\begin{tabular}{|c|c|c|c|}
\hline & $\begin{array}{l}\text { LAGC group }^{\mathrm{a})} \\
\quad(\mathrm{n}=\mathbf{1 4})\end{array}$ & $\begin{array}{l}\text { PAN group } \\
\quad(n=17)\end{array}$ & $\begin{array}{c}\text { Total } \\
(n=31)\end{array}$ \\
\hline Without surgery & $3(21.4)$ & 0 & $3(9.7)^{c)}$ \\
\hline With surgery & $11(78.6)$ & $17(100)$ & $28(90.3)$ \\
\hline $\mathrm{R} 0$ resection & $10(71.4)$ & $10(58.8)$ & $20(64.5)$ \\
\hline $\mathrm{R} 1 / 2$ resection & 0 & $4(23.5)$ & $4(12.9)$ \\
\hline $\mathrm{O} \& \mathrm{C}$ & $1(7.1)$ & $3(17.6)$ & $\left.4(12.9)^{d}\right)$ \\
\hline \multicolumn{4}{|l|}{ Pathological findings ${ }^{\text {e) }}$} \\
\hline Stage 0 (pCR) & $1(7.1)$ & $3(17.6)$ & $4(12.9)$ \\
\hline Stage IA & $1(7.1)$ & 0 & $1(3.2)$ \\
\hline Stage IB & $3(21.4)$ & $1(5.9)$ & $4(12.9)$ \\
\hline Stage IIA & $2(14.3)$ & 0 & $2(6.5)$ \\
\hline Stage IIB & $1(7.1)$ & $2(11.8)$ & $3(9.7)$ \\
\hline Stage IIIA & 0 & $1(5.9)$ & $1(3.2)$ \\
\hline Stage IIIB & 0 & $1(5.9)$ & $1(3.2)$ \\
\hline Stage IIIC & $2(14.3)$ & $2(11.8)$ & $4(12.9)$ \\
\hline Stage IV & 0 & $3(17.6)$ & $3(9.7)$ \\
\hline T0N1M0 & - & $1(5.9)$ & $1(3.2)$ \\
\hline Lymphovascular invasion & $5(35.7)$ & $6(35.3)$ & $11(35.5)$ \\
\hline Perineural invasion & $4(28.6)$ & $2(11.8)$ & $6(19.4)$ \\
\hline \multicolumn{4}{|l|}{ Lauren classification ${ }^{\mathrm{f})}$} \\
\hline Intestinal type & $7(50)$ & $8(47.1)$ & $15(48.4)$ \\
\hline Diffuse type & $7(50)$ & $7(41.2)$ & $14(45.2)$ \\
\hline Indeterminate & - & $2(11.8)$ & $2(6.5)$ \\
\hline
\end{tabular}

Values are presented as number (\%). LAGC, locally advanced gastric cancer; PAN, paraaortic lymph node; O\&C, opening

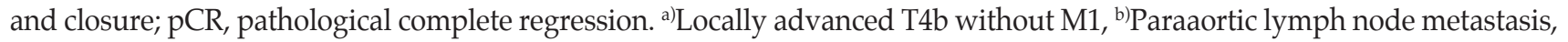

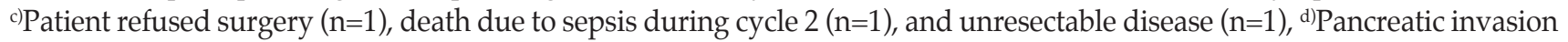
$(n=2)$, severe adhesion $(n=1)$, and peritoneal seeding $(n=1)$, e)Specimens analyzed from patients ( $n=24)$ who underwent gastrectomy, ${ }^{\mathrm{f}}$ Specimens $(\mathrm{n}=31)$ analyzed from surgical or endoscopic biopsy tissues.

\section{OS and PFS}

After a median follow-up of 44.5 months (range, 39.4 to 49.7 months), the median PFS and OS were 13.1 months $(95 \% \mathrm{CI}$, 8.9 to 17.3 ) and 38.6 months (95\% CI, 22.0 to 55.1), respectively (Fig. 1A and B). The LAGC group displayed trends toward increasing survival outcomes compared with the PAN group. The median PFS in the LAGC and PAN groups were 23.4 months (95\% CI, 3.0 to 43.8) and 12.2 months (95\% CI, 5.2 to 19.2), respectively $(p=0.327)$ (Fig. 1A). Furthermore, the median OS in the LAGC and PAN groups were 51.5 months (95\% CI, 37.1 to 65.9 ) and 19.3 months (95\% CI, 12.2 to 26.3), respectively ( $\mathrm{p}=0.138$ ) (Fig. $1 \mathrm{~B})$.

Patients who achieved $\mathrm{R} 0$ resection had significantly longer median PFS and OS than those who did not. The median PFS of patients who achieved $\mathrm{R} 0$ resection and those who did not were 23.4 months (95\% CI, 0.0 to 52.4) and 5.2 months (95\% CI, 3.7 to 6.6), respectively ( $\mathrm{p}<0.001)$ (Fig. 2A). Moreover, the median OS of patients who achieved R0 resection and those who did not were 51.5 months ( $95 \%$ CI, 28.6 to 74.4) and 19.3 months (95\% CI, 8.3 to 30.2), respectively ( $p=0.015$ ) (Fig. 2B). Trends toward increasing survival outcomes was also observed among patients with R0 resection in the LAGC group compared with those in the PAN group (median PFS, 38.6 vs. 14.1 months [ $\mathrm{p}=0.531$ ]; median OS, 51.5 vs. 25.3 months [ $\mathrm{p}=0.399]$, respectively) (Fig. 2A and B). Patients who achieved $\mathrm{R} 0$ resection had 2-year PFS and OS rates of $48 \%$ and $54 \%$, whereas those who did not had 2-year PFS and OS rates of $0 \%$ and $12 \%(\mathrm{p}<0.001$ and $\mathrm{p}=0.022$; data not shown), respectively.

The median PFS and OS were compared based on the pathological responses. The TRG groups were further categorized into two subgroups: TRG1 and TRG2-TRG5. Subsequently, we analyzed differences in the PFS and OS rates between the two subgroups. The TRG1 subgroup showed trends toward a higher PFS than the TRG2-TRG5 subgroup. 
A
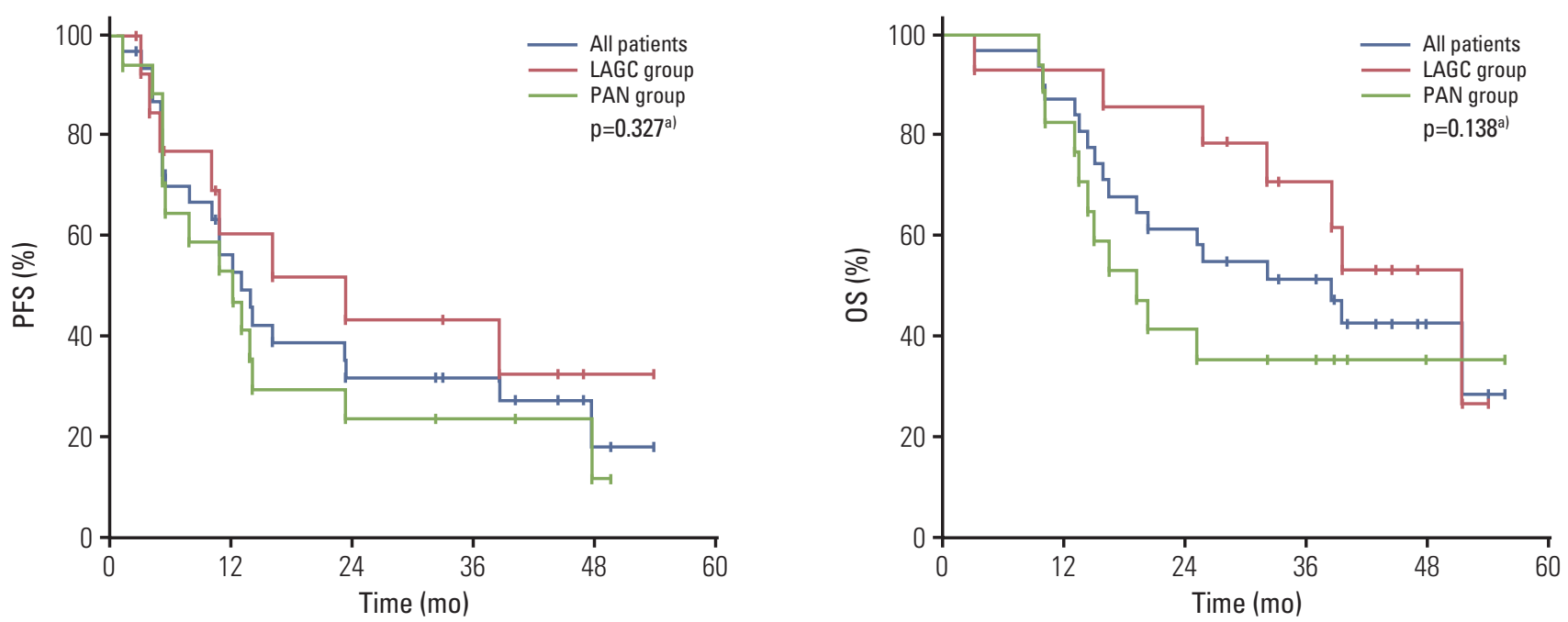

Fig. 1. Kaplan-Meier curves for progression-free survival (PFS) (A) and overall survival (OS) (B) in all patients and groups categorized based on the causes of initial unresectability. The locally advanced gastric cancer (LAGC) group displayed a trend toward better survival outcomes compared with the paraaortic lymph node (PAN) group (median PFS, 23.4 months vs. 12.2 months, [ $\mathrm{p}=0.327$ ]; median OS, 51.5 months vs. 19.3 months [ $\mathrm{p}=0.138]$, respectively). ${ }^{\mathrm{a}}$ Compared with LAGC group.
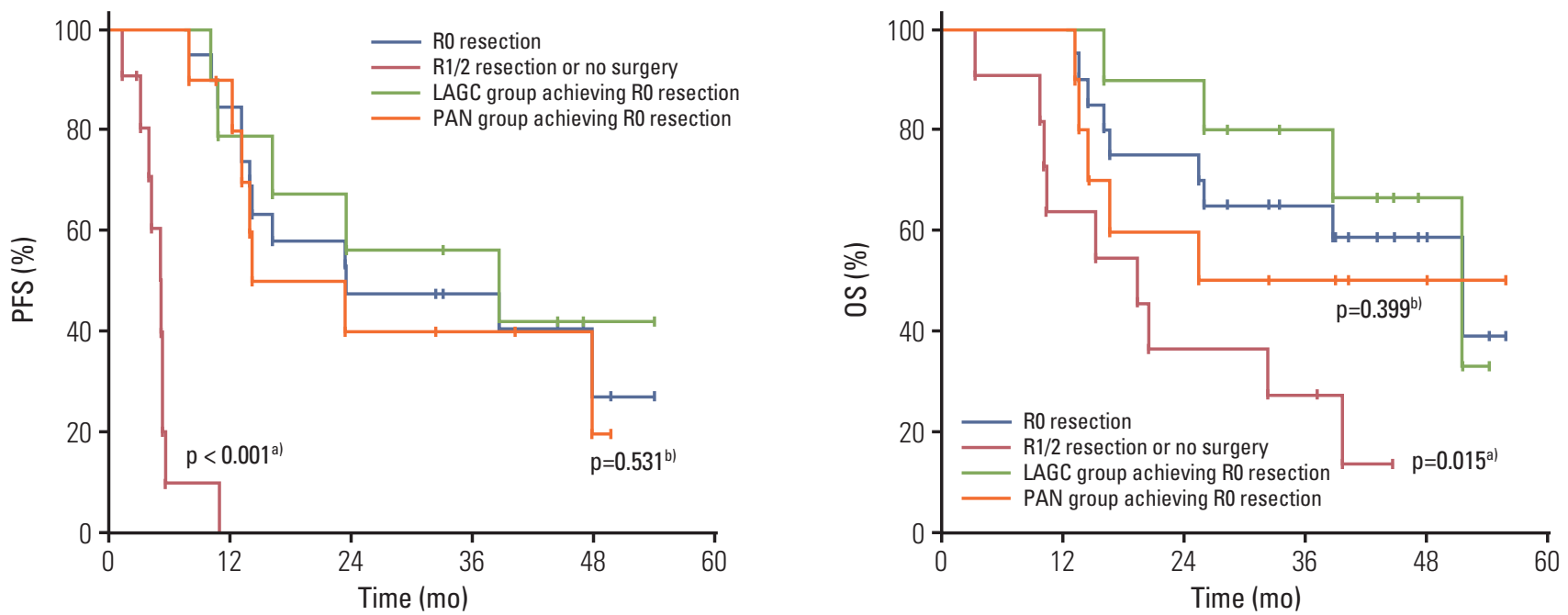

Fig. 2. Kaplan-Meier curves for progression-free survival (PFS) (A) and overall survival (OS) (B) based on R0 resectability. Patients who achieved R0 resection had significantly longer median PFS and OS than those who did not (median PFS, 23.4 months vs. 5.2 months [p < 0.001]; median OS, 51.5 months vs. 19.3 months [p=0.015], respectively). PFS (A) and OS (B) of patients with R0 resection were classified based on the causes of initial unresectability. The locally advanced gastric cancer (LAGC) group showed a trend toward better survival outcomes than the paraaortic lymph node (PAN) group (median PFS, 38.6 vs. 14.1 months [ $\mathrm{p}=0.531$ ]; median OS, 51.5 vs. 25.3 months [ $\mathrm{p}=0.399$ ], respectively). a)Compared with R0 resection, ${ }^{\mathrm{b})}$ Compared with LAGC group achieving R0 resection. 
A
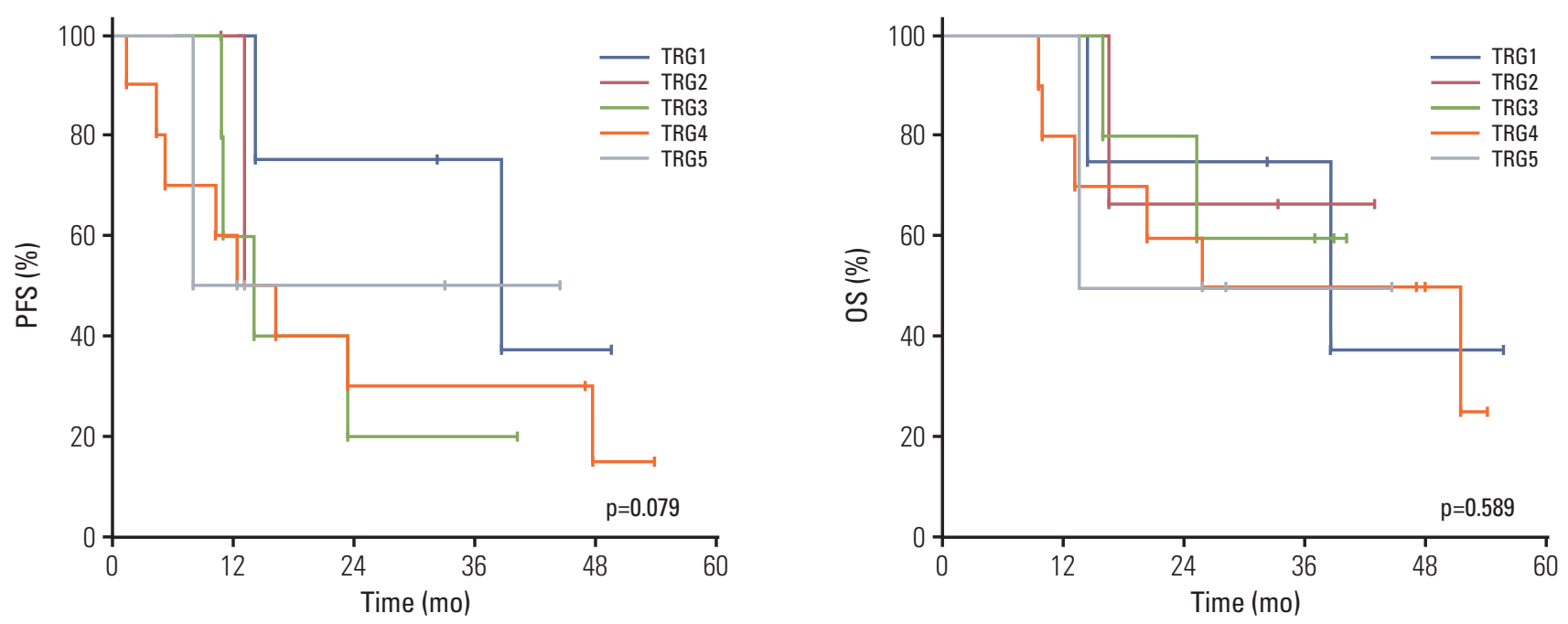

C
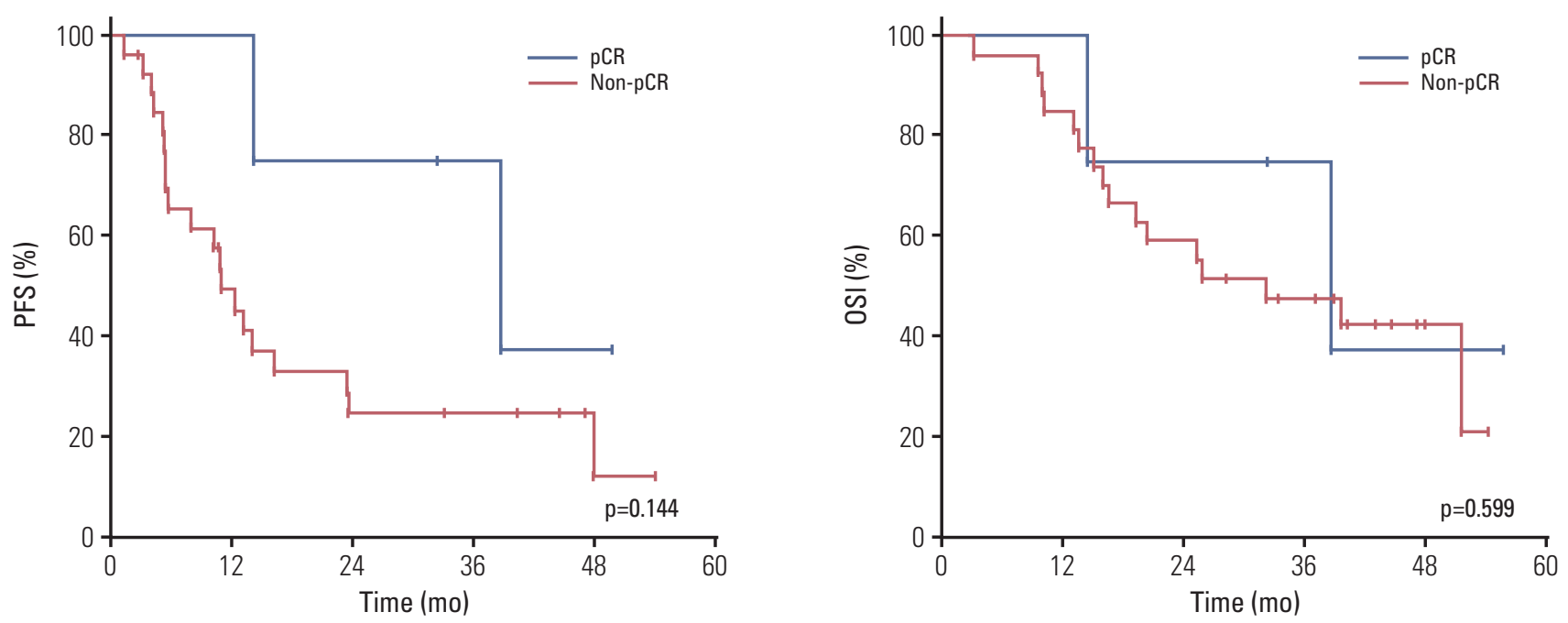

Fig. 3. Kaplan-Meier curves for progression-free survival (PFS) (A) and overall survival (OS) (B) based on the tumor regression grades using log-rank test between two subgroups: tumor regression grade (TRG) 1 and TRG2-TRG5. Kaplan-Meier curves for PFS (C) and OS (D) with respect to pathological complete regression (pCR).

However, this finding was not observed in terms of OS ( $\mathrm{p}=0.079$ and $\mathrm{p}=0.589$, respectively) (Fig. $3 \mathrm{~A}$ and B). Patients with $\mathrm{pCR}$ showed trends toward longer PFS than those without pCR (38.6 months vs. 10.8 months, $\mathrm{p}=0.144$ ). Additionally, no significant difference was observed in the OS between patients with pCR and those without (38.6 months vs. 32.2 months, $\mathrm{p}=0.599$ ) (Fig. 3C and D).

\section{Failure pattern}

Recurrence was observed in 12 out of 20 patients ( $60 \%$ ) who achieved $\mathrm{R} 0$ resection. Locoregional recurrence $(\mathrm{n}=2)$ and peritoneal seeding $(n=2)$ were the modes of recurrence in the LAGC group (four out of 10 patients with R0 resection, $40 \%$ ). On the contrary, locoregional recurrence $(n=3)$, extra-abdominal LN metastases $(n=2)$, peritoneal seeding $(n=1)$, colon invasion $(n=1)$, and brain invasion $(n=1)$ were the modes of 
recurrence observed in the PAN group (8 out of 10 patients with $\mathrm{R} 0$ resection, $80 \%$ ).

\section{Discussion}

Our results showed that induction chemotherapy with DXP plus BEV exerted antitumor activity in patients with unresectable LAGC or PAN metastatic GC, which led to the possibility of curative surgery in approximately two-thirds of patients. Patients who achieved R0 resection showed significantly improved survival outcomes in terms of median PFS and OS as well as 2-year PFS and OS compared with those who did not. Patients with $\mathrm{pCR}$, which was considered as a highly valuable prognostic factor [20], also had higher PFS rate and showed signs of better antitumor activity than those who did not. Additionally, two patients remained alive without evidence of disease (OS, 55.8 and 32.3 months, respectively).

Considering that curative surgery is the most important prognostic factor in GC, upfront surgery followed by chemotherapy for GC with a single metastasis or conversion surgery following systemic chemotherapy for initially unresectable GC is considered. A recent randomized trial (Reductive Gastrectomy for Advanced Tumor in Three Asian countries, REGATTA) evaluating the role of upfront surgery in patients who had GC with a single noncurable factor confined to either the liver (H1), peritoneum (P1), or PAN $(16 \mathrm{a} 1 / \mathrm{b} 2)$ failed to show the survival benefit of gastrectomy followed by chemotherapy compared with chemotherapy alone [21]. However, our previous study on the effect of induction chemotherapy with DXP on unresectable GC as a result of local invasion or limited intraabdominal metastasis in the PAN and/or peritoneum suggested that this chemotherapeutic regimen led to excellent possibility for curative surgery in patients with local invasion (R0 resection rate, $71 \%$ ) or PAN metastasis (70\%); however, patients with peritoneal metastasis were less likely to receive curative surgery $(36 \%)$ than those without [12]. These findings suggest that patients with PAN metastasis could be suitable candidates for induction chemotherapy followed by conversion surgery [12].

BEV was combined with a triplet regimen in our study to intensify the induction chemotherapy strategy. Although the preferences for doublet versus triplet regimens vary among geographical regions, the latter has been suggested to have better antitumor activity than the former, as shown by the results of the V325 trial, which compared cisplatin+5-fluorouracil (CF) with docetaxel+CF as a first-line therapy for AGC [22]. We previously showed the promising efficacy of the DXP as induction and palliative chemotherapy regimen [23]. A phase III AVAGAST study revealed significantly improved PFS (median, 6.7 months vs. 5.3 months; $\mathrm{p}=0.0037$ ) and ORR ( $46.0 \%$ vs. $37.4 \%, \mathrm{p}=0.0315)$ with the administration of BEV plus chemotherapy [15], indicating that the addition of BEV to chemotherapeutic regimen led to enhanced antitumor effects. BEV has been also widely combined with firstline chemotherapeutic regimens for the treatment of metastatic colorectal cancer, resulting in improved PFS and OS [24]. Although the effect of BEV on unresectable colorectal cancer remains unclear, an irinotecan-based regimen with BEV appears to be effective in converting unresectable to resectable disease with high ORR [24]. Furthermore, two recent phase III studies demonstrated that ramucirumab (monoclonal antibody against vascular endothelial growth factor receptor 2 [VEGFR2]) alone or combined with another chemotherapy regimen significantly improved survival among patients with AGC in the second-line setting $[25,26]$. Apatinib, a tyrosine kinase inhibitor against VEGFR2, was also found to have survival benefits among patients in the third-line setting [27]. These results suggest that a strategy involving antiangiogenic drugs is effective in treating GC.

However, in the present study, DXP combined with BEV did not appear to improve the treatment outcomes in terms of R0 resection rate, unlike the finding of a previous phase II study that used DXP alone. The results showed R0 resection rates of $71 \%$ and $71.4 \%$ for DXP alone and DXP plus BEV, respectively, in locally advanced disease and $70 \%$ and $58.8 \%$ for DXP alone and DXP plus BEV, respectively, in PAN metastatic disease [12]. In a recent phase III study (Medical Research Council Adjuvant Gastric Infusional Chemotherapy [MAGIC]-B/ST03), perioperative BEV plus chemotherapy did not result in OS (hazard ratio [HR], 1.067; $\mathrm{p}=0.478$ ), PFS (HR, 1.026; $\mathrm{p}=0.768$ ), or disease-free survival (HR, 1.006; $\mathrm{p}=0.942$ ) benefits compared with chemotherapy alone in resectable GC [28]. Differences in R0 resection, clinical response, and $\mathrm{PCR}$ were also not observed between chemotherapy alone and chemotherapy plus BEV (75\% vs. $76 \%$, $42 \%$ vs. $40 \%$, and $8 \%$ vs. $10 \%$, respectively) [28]. The addition of $\mathrm{BEV}$ to the chemotherapeutic regimen in the preoperative setting did not lead to improved antitumor effects. However, interpretations of these data require caution with respect to the different baseline characteristics compared with the outcomes of our previous DXP study [12] and the combination of chemotherapeutic agents (e.g., epirubicin, cisplatin, and capecitabine regimen) used with $\mathrm{BEV}$, which may explain the differences in the antitumor effects and survival outcomes. In a recent randomized controlled study that involved patients who had LAGC with T3 or T4 clinical stages and PAN metastasis, induction chemotherapy with docetaxel, oxaliplatin, and fluorouracil (DOF) plus BEV improved the ORR, R0 resection, and median PFS compared with the DOF 
regimen alone (ORR, 65\% vs. $42.5 \%$ [ $\mathrm{p}=0.043$ ]; $\mathrm{R} 0$ resection rate, $75 \%$ vs. $50 \%$ [p=0.020]; and median PFS, 15.2 vs. 12.3 months [ $\mathrm{p}=0.01]$ ) [29]. Docetaxel (a taxane) may be preferably combined with BEV rather than epirubicin (an anthracycline drug) because the antiangiogenic property of the former is synergistic with that of VEGF inhibitors [30]. The role of antiangiogenesis in the preoperative setting requires further investigation.

Although the triplet regimen appears to produce better antitumor effects than doublet regimen, the use of the former is a constant cause of concern [22]. In a previous study, induction chemotherapy with DXP regimen resulted in hematologic toxicity as a major adverse effect. Furthermore, grade $3 / 4$ neutropenia was reported in $69 \%$ of the patients, although febrile neutropenia only occurred in $4 \%$ of the participants [12]. In the present study, the DXP plus BEV regimen led to a marginally higher grade $3 / 4$ neutropenia incidence $(74.2 \%)$ than DXP alone. The higher grade $3 / 4$ neutropenia incidence may be attributed to the frequent $\mathrm{CBC}$ measurement, which was performed every week to monitor the trend toward decreasing neutrophil counts. Although the present study showed considerably higher incidence of grade $3 / 4$ febrile neutropenia $(22.6 \%$ ) than the previous studies, the majority of adverse events did not necessitate hospitalization, and the neutrophil counts of the patents returned to normal levels as well. This higher febrile neutropenia incidence may be related to the higher stomatitis incidence (grade $3 / 4 ; 16.1 \%$ vs. $2 \%$ for DXP plus BEV and DXP, respectively) because disruption of the mucosal barrier may cause the patients to become susceptible to infection. Although another toxicity profile in our study showed similarities between the use of DXP alone and that of DXP plus BEV, the latter resulted in BEV-related toxicities, including grade 3 gastric perforation $(9.7 \%)$, grade 3 pulmonary thromboembolism $(3.2 \%)$, and grade 1 hypertension (3.2\%). The BEV- related toxicities detected in our study were comparable to those observed in a previous trial. In the MAGIC-B/ST03 study, chemotherapy combined with BEV led to increased postoperative anastomotic leak incidence compared with chemotherapy alone (18\% vs. $9 \%$, respectively), specifically in patients who underwent esophagogastrostomy (9\% vs. $23 \%$, respectively) [28]. However, our study did not show any increase in major surgical complications with the use of $\mathrm{BEV}$, which may be caused by the differences in the tumor location and surgical technique used from those of the MAGIC-B / ST03 study.

Induction chemotherapy with DXP plus BEV demonstrated promising antitumor efficacy with encouraging curative resection rates and long-term survival outcomes. Further studies are warranted to investigate the optimal strategy for induction chemotherapy followed by conversion surgery and identify the population that is most likely to benefit from this strategy.

\section{Conflicts of Interest}

Yoon-Koo Kang is a consultant for F. Hoffmann-La Roche AG, Novartis International AG, Sanofi S.A., Ono Pharmaceutical Co. Ltd., Daehwa Pharma Co. Ltd., Bristol-Myers Squibb, AstraZeneca, and Blueprint Medicines. Sook Ryun Park and Jihun Kim has received speaker honorarium from F. Hoffmann-La Roche AG and Merck \& Co. Inc., respectively. The authors have no other conflicts of interest to declare.

\section{Acknowledgments}

This work was supported by the Asan Medical Center, Republic of Korea. Capecitabine and bevacizumab were provided by Roche Korea Ltd.

\section{References}

1. Jung KW, Won YJ, Kong HJ, Oh CM, Cho H, Lee DH, et al. Cancer statistics in Korea: incidence, mortality, survival, and prevalence in 2012. Cancer Res Treat. 2015;47:127-41.

2. Koo DH, Ryu MH, Ryoo BY, Seo J, Lee MY, Chang HM, et al. Improving trends in survival of patients who receive chemotherapy for metastatic or recurrent gastric cancer: 12 years of experience at a single institution. Gastric Cancer. 2015;18: 346-53.

3. Smalley SR, Benedetti JK, Haller DG, Hundahl SA, Estes NC, Ajani JA, et al. Updated analysis of SWOG-directed intergroup study 0116: a phase III trial of adjuvant radiochemotherapy versus observation after curative gastric cancer resection. J Clin Oncol. 2012;30:2327-33.

4. Cunningham D, Allum WH, Stenning SP, Thompson JN, Van de Velde CJ, Nicolson M, et al. Perioperative chemotherapy versus surgery alone for resectable gastroesophageal cancer. N Engl J Med. 2006;355:11-20.

5. Ychou M, Boige V, Pignon JP, Conroy T, Bouche O, Lebreton $\mathrm{G}$, et al. Perioperative chemotherapy compared with surgery alone for resectable gastroesophageal adenocarcinoma: an FNCLCC and FFCD multicenter phase III trial. J Clin Oncol. 2011;29:1715-21. 
6. Sakuramoto S, Sasako M, Yamaguchi T, Kinoshita T, Fujii M, Nashimoto A, et al. Adjuvant chemotherapy for gastric cancer with S-1, an oral fluoropyrimidine. N Engl J Med. 2007;357: 1810-20.

7. Bang YJ, Kim YW, Yang HK, Chung HC, Park YK, Lee KH, et al. Adjuvant capecitabine and oxaliplatin for gastric cancer after D2 gastrectomy (CLASSIC): a phase 3 open-label, randomised controlled trial. Lancet. 2012;379:315-21.

8. Fukuchi M, Ishiguro T, Ogata K, Suzuki O, Kumagai Y, Ishibashi $\mathrm{K}$, et al. Prognostic role of conversion surgery for unresectable gastric cancer. Ann Surg Oncol. 2015;22:3618-24.

9. Du Y, Yu P. Conversion chemotherapy combined with surgical treatment of unresectable advanced gastric cancer. J Clin Oncol. 2015;33(3 Suppl):Abstr 189.

10. Tsuburaya A, Mizusawa J, Tanaka Y, Fukushima N, Nashimoto A, Sasako M, et al. Neoadjuvant chemotherapy with S-1 and cisplatin followed by D2 gastrectomy with paraaortic lymph node dissection for gastric cancer with extensive lymph node metastasis. Br J Surg. 2014;101:653-60.

11. Inoue K, Nakane Y, Kogire M, Fujitani K, Kimura Y, Imamura $\mathrm{H}$, et al. Phase II trial of preoperative S-1 plus cisplatin followed by surgery for initially unresectable locally advanced gastric cancer. Eur J Surg Oncol. 2012;38:143-9.

12. Sym SJ, Chang HM, Ryu MH, Lee JL, Kim TW, Yook JH, et al. Neoadjuvant docetaxel, capecitabine and cisplatin (DXP) in patients with unresectable locally advanced or metastatic gastric cancer. Ann Surg Oncol. 2010;17:1024-32.

13. Shah MA, Jhawer M, et al. Ilson DH, Lefkowitz RA, Robinson E, Capanu M, et al. Phase II study of modified docetaxel, cisplatin, and fluorouracil with bevacizumab in patients with metastatic gastroesophageal adenocarcinoma. J Clin Oncol. 2011;29:868-74.

14. Shah MA, Ramanathan RK, Ilson DH, Levnor A, D'Adamo D, O'Reilly E, et al. Multicenter phase II study of irinotecan, cisplatin, and bevacizumab in patients with metastatic gastric or gastroesophageal junction adenocarcinoma. J Clin Oncol. 2006;24:5201-6.

15. Ohtsu A, Shah MA, Van Cutsem E, Rha SY, Sawaki A, Park $\mathrm{SR}$, et al. Bevacizumab in combination with chemotherapy as first-line therapy in advanced gastric cancer: a randomized, double-blind, placebo-controlled phase III study. J Clin Oncol. 2011;29:3968-76.

16. Colevas AD, Setser A. The NCI Common Terminology Criteria for Adverse Events (CTCAE) v 3.0 is the new standard for oncology clinical trials. J Clin Oncol. 2004;22(14 Suppl):Abstr 6098.

17. Therasse P, Arbuck SG, Eisenhauer EA, Wanders J, Kaplan RS, Rubinstein L, et al. New guidelines to evaluate the response to treatment in solid tumors. European Organization for Research and Treatment of Cancer, National Cancer Institute of the United States, National Cancer Institute of Canada. J Natl Cancer Inst. 2000;92:205-16.

18. Koh YW, Park YS, Ryu MH, Ryoo BY, Park HJ, Yook JH, et al. Postoperative nodal status and diffuse-type histology are independent prognostic factors in resectable advanced gastric carcinomas after preoperative chemotherapy. Am J Surg
Pathol. 2013;37:1022-9.

19. Mandard AM, Dalibard F, Mandard JC, Marnay J, HenryAmar M, Petiot JF, et al. Pathologic assessment of tumor regression after preoperative chemoradiotherapy of esophageal carcinoma: clinicopathologic correlations. Cancer. 1994;73:2680-6.

20. Becker K, Langer R, Reim D, Novotny A, Meyer zum Buschenfelde C, Engel J, et al. Significance of histopathological tumor regression after neoadjuvant chemotherapy in gastric adenocarcinomas: a summary of 480 cases. Ann Surg. 2011;253: 934-9.

21. Fujitani K, Yang HK, Mizusawa J, Kim YW, Terashima M, Han $\mathrm{SU}$, et al. Gastrectomy plus chemotherapy versus chemotherapy alone for advanced gastric cancer with a single non-curable factor (REGATTA): a phase 3, randomised controlled trial. Lancet Oncol. 2016;17:309-18.

22. Van Cutsem E, Moiseyenko VM, Tjulandin S, Majlis A, Constenla M, Boni C, et al. Phase III study of docetaxel and cisplatin plus fluorouracil compared with cisplatin and fluorouracil as first-line therapy for advanced gastric cancer: a report of the V325 Study Group. J Clin Oncol. 2006;24:4991-7.

23. Kang YK, Ryu MH, Yoo C, Chang HM, Yook JH, Oh ST, et al. Phase I/II study of a combination of docetaxel, capecitabine, and cisplatin (DXP) as first-line chemotherapy in patients with advanced gastric cancer. Cancer Chemother Pharmacol. 2011;67:1435-43.

24. Hurwitz H, Fehrenbacher L, Novotny W, Cartwright T, Hainsworth J, Heim W, et al. Bevacizumab plus irinotecan, fluorouracil, and leucovorin for metastatic colorectal cancer. N Engl J Med. 2004;350:2335-42.

25. Fuchs CS, Tomasek J, Yong CJ, Dumitru F, Passalacqua R, Goswami C, et al. Ramucirumab monotherapy for previously treated advanced gastric or gastro-oesophageal junction adenocarcinoma (REGARD): an international, randomised, multicentre, placebo-controlled, phase 3 trial. Lancet. 2014;383: 31-9.

26. Wilke H, Muro K, Van Cutsem E, Oh SC, Bodoky G, Shimada $Y$, et al. Ramucirumab plus paclitaxel versus placebo plus paclitaxel in patients with previously treated advanced gastric or gastro-oesophageal junction adenocarcinoma (RAINBOW): a double-blind, randomised phase 3 trial. Lancet Oncol. 2014;15:1224-35.

27. Qin S. Phase III study of apatinib in advanced gastric cancer: a randomized, double-blind, placebo-controlled trial. J Clin Oncol. 2014;32(15 Suppl):Abstr 4003.

28. Cunningham D, Smyth E, Stenning S, Stevenson L, Robb C, Allum $W$, et al. Peri-operative chemotherapy \pm bevacizumab for resectable gastro-oesophageal adenocarcinoma: results from the UK Medical Research Council randomised ST03 trial (ISRCTN 46020948). Eur J Cancer. 2015;51 Suppl 3:Abstr 2201.

29. Ma J, Yao S, Li XS, Kang HR, Yao FF, Du N. Neoadjuvant therapy of DOF regimen plus bevacizumab can increase surgical resection ratein locally advanced gastric cancer: a randomized, controlled study. Medicine (Baltimore). 2015;94:e1489.

30. Sweeney CJ, Miller KD, Sissons SE, Nozaki S, Heilman DK, Shen J, et al. The antiangiogenic property of docetaxel is syn- 
ergistic with a recombinant humanized monoclonal antibody against vascular endothelial growth factor or 2-methoxyestra- diol but antagonized by endothelial growth factors. Cancer Res. 2001;61:3369-72. 